\title{
Isotropic-Liquid Crystal Phase Equilibrium in Aqueous Solutions of a Triple-Helical Polysaccharide Schizophyllan
}

\author{
Kazuo VAN and Akio Teramoto \\ Department of Macromolecular Science, Osaka University, \\ Toyonaka, Osaka 560, Japan
}

(Received September 16, 1982)

\begin{abstract}
A polysaccharide schizophyllan known to have a rodlike shape in solution was investigated. The system schizophyllan + water exhibited an equilibrium between isotropic and cholesteric phases. The temperature-concentration phase diagram in a temperature range $5-80^{\circ} \mathrm{C}$ and a concentration range $0-38.8$ polymer $\mathrm{wt} \%$ was determined. The phase diagram determined is characterized by a narrow biphasic region separating the isotropic phase from the cholesteric phase and resembles those reported by Miller and coworkers for polypeptide solutions, except that it has no broad biphasic region at low temperature. The experimental results for schizophyllan appear to favor the Flory-Ronca theory of rodlike molecules, but the data are not sufficiently detailed to. draw definite conclusions.

KEY WORDS Cholesteric Liquid Crystal / Phase Equilibrium / Phase Diagram / Polysaccharide / Schizophyllan / Rodlike Molecule / Polarizing Microscopy / Laser Light Diffraction /
\end{abstract}

In a previous paper ${ }^{1}$ we showed that a $\beta-(1-3)-\mathrm{D}-$ glucan schizophyllan in aqueous solution forms a cholesteric mesophase at a polymer concentration higher than $10 \mathrm{wt} \%$. At intermediate concentrations above $10 \%$, there exists an equilibrium between isotropic and cholesteric phases, which shifts somewhat with temperature. The cholesteric phase shows characteristic fingerprint patterns with the cholesteric pitch decreasing markedly with increasing polymer concentration.

The present study takes up this phase equilibrium in greater detail. The system schizophyllan + water was chosen again, because (1) in aqueous solution, schizophyllan exists in the form of triple-stranded helix which can be represented by a uniform cylinder, $;^{2-4}$ this well-characterized helical conformation allows us to test theories of rodlike polymer solutions, (2) schizophyllan dissolves in aqueous media thus facilitating various physical measurements, and (3) samples in a wide range of molecular weight are available. ${ }^{2-4}$ This good solubility distinguishes schizophyllan from other rodlike polymers which form mesophases but usually have limited solubility. ${ }^{5-7}$ Xanthan, an ionizable poly- saccharide, is one of a few mesomorphic polymers having good solubility. ${ }^{8}$

In order to obtain the temperature-concentration phase diagram, two new methods were applied to the present system: one using the concentration dependence of the cholesteric pitch as a function of temperature and the other, a sedimentation analysis of solutions consisting of isotropic and cholesteric phases in equilibrium. The former method was effectively used for determining the boundary between the biphasic and cholesteric regions, i.e., the B-point, which, otherwise, was not easy to be determined accurately. ${ }^{9-11}$ In the course of the present study, we noted that sedimentation analysis had been used for similar purposes. ${ }^{12-14}$

\section{EXPERIMENTAL}

Schizophyllan sample D-40 used in our previous study ${ }^{1}$ had a weight-average molecular weight $M_{w}$ of 478000 , with an $M_{z} / M_{w}$ of 1.2 , where $M_{z}$ is the $z$-average molecular weight. This was again used throughout the present investigation.

Polymer solutions were prepared by mixing re- 
quired amounts of sample D-40 and water in stoppered flasks. The polymer weight fraction $w_{2}$ in the solution was determined gravimetrically and the polymer mass concentration $c_{2}\left(\mathrm{~g} \mathrm{~cm}^{-3}\right)$ was calculated from $w_{2}$ and the specific volume $v\left(\mathrm{~cm}^{3} \mathrm{~g}^{-1}\right)$ of aqueous schizophyllan solutions at $25^{\circ} \mathrm{C}$ :

$$
v=1.0030-0.383 w_{2}+0.2044 w_{2}{ }^{2}-0.2609 w_{2}{ }^{3}
$$

Polarized light microscopy was performed in a Union Mec-3 microscope equipped with a thermostatting cell holder. Polymer solutions filled in drum-shaped cells were examined between crossed polars and by laser light diffraction at different temperatures between 5 and $80^{\circ} \mathrm{C}$. From these measurements the cholesteric pitch $2 \mathrm{~S}$ of the solutions was obtained as a function of temperature and polymer concentration. The detailed experimental procedures and the methods for data analysis are given in the previous paper. ${ }^{1}$

Sedimentation experiments were conducted in a Beckman-Spinco Model E analytical ultracentrifuge with Kel-F cells. In a phase separation experiment, an appropriate quantity of a polymer solution was placed in a Kel-F cell and the whole cell assembly was kept at $30^{\circ} \mathrm{C}$ at least for two days to effect phase separation. The cell assembly was then loaded on to a J-rotor heated at the same temperature and centrifuged at a rotor speed between 1500 and $5600 \mathrm{rpm}$. The phase separation was monitored by a schlieren optical system, the temperature of the cell being kept at $30.0 \pm 0.1^{\circ} \mathrm{C}$. Sedimentation equilibrium experiments on dilute solutions were performed at $25^{\circ} \mathrm{C}$ according to the standard procedure. ${ }^{2}$

\section{RESULTS}

\section{Cholesteric Pitch}

Schizophyllan solutions filled in drum-shaped cells were kept at $30^{\circ} \mathrm{C}$ for at least one week and examined by polarizing microscopy and laser light diffraction, first at $30^{\circ} \mathrm{C}$. Those solutions with $w_{2}$ above the B-point ( $c a .0 .13$ ) were strongly birefringent exhibiting patterns with alternate bright and dark parallel lines characteristic of a cholesteric mesophase. Such fingerprint patterns developed gradually throughout the entire solution within one week after placing the solution in the cell. The distance between the parallel lines $S$, half the cholesteric pitch, was found to be constant except in the

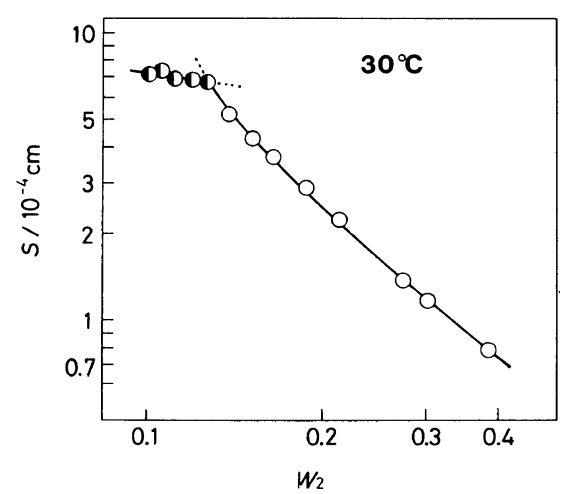

Figure 1. Plot of $S$ vs. $w_{2}$ for schizophyllan sample D40 in water at $30^{\circ} \mathrm{C}$. Open circles, the cholesteric region $\left(w_{2}\right)$; half-filled circles, the biphasic region $\left(w_{2}^{0}\right)$.

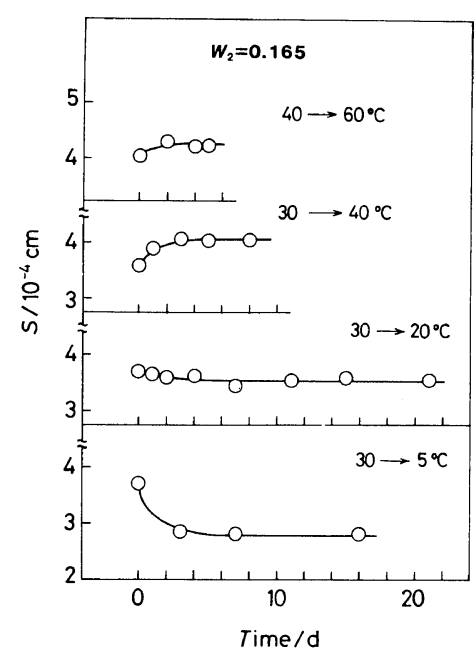

Figure 2. Variation in $S$ with the time elapsed after the temperature jump for sample D-40, $w_{2}=0.165$.

regions near the cell walls.

When illuminated by laser light without polars, the solutions showed diffraction rings, in most cases, of the first order only. Following the previous procedure, ${ }^{1}$ the $S$ of the solutions were determined as functions of $w_{2}$ from such diffraction rings as well as the photomicrographs of the fingerprint patterns. The results are displayed in Figure 1 by open circles.

Solutions with the overall weight fraction $w_{2}{ }^{0}$ between 0.0975 and 0.127 appeared biphasic and displayed no well-defined diffraction patterns. When a solution with $w_{2}{ }^{0}$ not too close to the boundary values was left standing at a fixed temperature, e.g., $30^{\circ} \mathrm{C}$, it separated into two liquid 


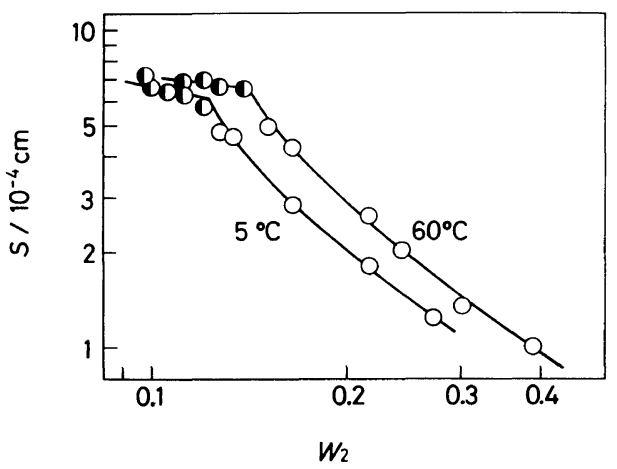

Figure 3. Plots of $S$ vs. $w_{2}$ for sample D-40 at 5 and $60^{\circ} \mathrm{C}$.

layers: one filled with fingerprint patterns and the other containing many spherulites or small regions of fingerprint patterns buried in a dark area of the isotropic phase. The $S$ value for such a biphasic mixture was obtained from the photomicrographs of the fingerprint patterns. Figure 1 shows the results for biphasic mixtures by plotting $S v s . w_{2}{ }^{0}$ as half-filled circles. It can be seen that the entire $S v s$. $w_{2}$ relationship is represented by a smooth curve with a break point. It should be remarked that complete separation into the two phases never occurred on standing but was possible only by centrifugation as will be illustrated below. This finding has previously been reported by other investigators. $^{12-14}$

Solutions containing a liquid crystal phase had viscosities higher than $5 \mathrm{~kg} \mathrm{~m}^{-1} \mathrm{~s}^{-1}$ at $30^{\circ} \mathrm{C}$. Thus it seemed that when the temperature was changed, it would take some time before the liquid crystal phase adjusted itself to a new equilibrium state. This time effect was examined by measuring $S$ as a function of the time elapsed after the temperature jump. Figure 2 illustrates typical results with $w_{2}=$ 0.165 , where the arrows indicate the changes in temperature. From these and similar results at other concentrations, we conclude that the values of $S$ after three days may be regarded as the equilibrium values.

Figure 3 shows the data for $S$ thus obtained at 5 and $60^{\circ} \mathrm{C}$. Similar data were obtained at 20,40 and $80^{\circ} \mathrm{C}$. After being examined at these temperatures, each solution was examined at $30^{\circ} \mathrm{C}$, and it was found that $S$ was the same within $\pm 3 \%$ before and after the heating cycle. Thus, we see that $S$ is a unique function of temperature $T$ and polymer weight fraction $w_{2}$ for a given schizophyllan sam- ple.

\section{Phase Diagram}

The results for $S$ presented above show that $S$ is substantially constant in the biphasic region. This trend may be anticipated if the solution separates into two phases of essentially fixed concentrations irrespective of the overall concentration. On the other hand, $S$ is expected to change monotonically with $w_{2}$ in the liquid crystal region. This is because $S$ is essentially determined by the concentration of the cholesteric phase, regardless of whether the phase is in a pure cholesteric solution or in a biphasic mixture. Thus the break point of each $S v s . w_{2}$ curve can be identified as the boundary between the liquid crystal and biphasic regions, i.e., the B-point. In this way, the phase boundary curve extending from 5 to $80^{\circ} \mathrm{C}$ was determined from these and similar results at other temperatures, as shown in Figure 4, where horizontal segments indicate the uncertainty associated with the graphical determination of the phase boundary concentration. It can be seen that the cholesteric liquid crystal region extends at least up to $38.8 \mathrm{wt} \%$.

The boundary between the isotropic and biphasic regions, i.e., the A-point, was determined as follows. First, a solution with an overall concentration $w_{2}{ }^{0}$ as prepared or stored for a long time period at a specified temperature $T$, was examined by polarizing microscopy so as to prove it to be isotropic. This set of $w_{2}{ }^{0}$ and $T$ gives one data point in the isotropic region and is indicated by the filled circle in Figure 4. Next, a slightly more concentrated solution was examined at the same temperature to prove it to be birefringent. This set gives another data point for the same temperature in the biphasic region and is represented by the open circle. The phase boundary at $T$ can thus be located between these two data points.

The existence of a temperature-induced isotropiccholesteric phase transition was found as follows. The solution with $w_{2}{ }^{0}=0.0975$ was biphasic at $30^{\circ} \mathrm{C}$ as prepared and had an $S$ value close to that for the B-point at the same temperature. When the temperature was raised, it became progressively isotropic. It was observed that some regions of the solution resisted more strongly higher temperature than others, and the final traces of the cholesteric phase remained up to a temperature as high as $55^{\circ} \mathrm{C}$. When cooled, the solution still remained 


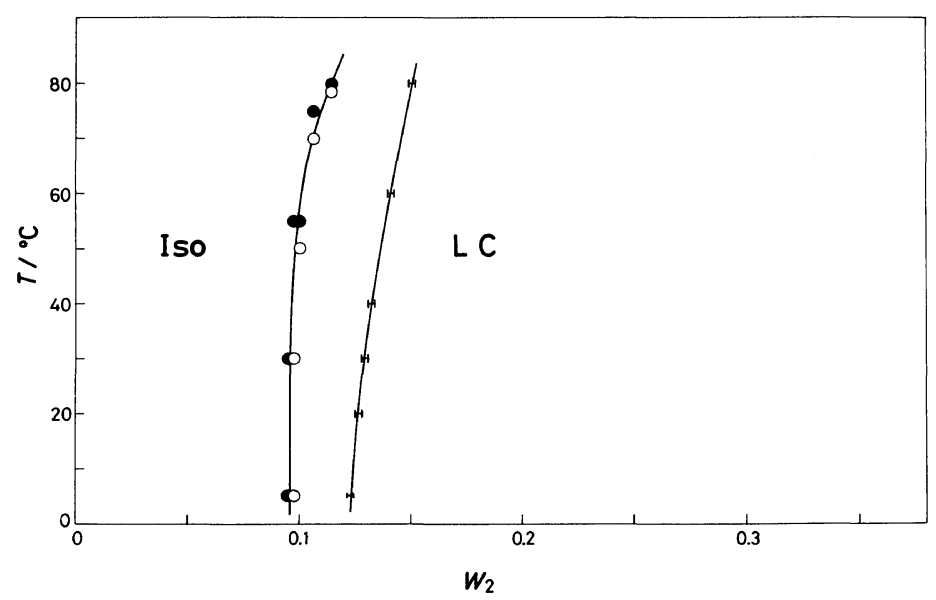

Figure 4. Temperature-concentration phase diagram for the system sample D-40+water. Horizontal segments, uncertainties associated with the graphical determination; filled circles, the isotropic region; open circles, the biphasic region. Here $w_{2}$ in the biphasic region stands for $w_{2}{ }^{0}$.

isotropic at $40^{\circ} \mathrm{C}$ but was found distinctly biphasic again at $30^{\circ} \mathrm{C}$. Thus the phase boundary temperature should be around $40^{\circ} \mathrm{C}$ but no precise determination could be made.

Similar experiments were conducted with other solutions. These results are also included in Figure 4. It can be seen that the higher the overall concentration, the smaller the gap between the open and filled circles for the same $w_{2}{ }^{0}$. The solid curve drawn through the gaps between the circles separates the isotropic region from the biphasic region. In spite of the large temperature gaps at lower $w_{2}{ }^{0}$, the phase boundary curve could be determined with reasonable accuracy, since it was almost vertical.

\section{Sedimentation Analysis}

When a solution in the biphasic region was centrifuged at a rotor speed above $2000 \mathrm{rpm}$, it separated into two distinct layers. The isotropic phase appeared on top of the cholesteric phase with a wide meniscus intervening them. In many cases, either or both of the phases exhibited concentration gradient curves. The volume of the isotropic phase, $\Phi$, relative to that of the total solution was determined from the column height of this phase relative to the solution height.

It was found that $\Phi$ increased slightly with increasing rotor speed, indicating that the centrifugal force had some effect on the phase equilibrium. The values of $\Phi$ at rotor speeds between 2000 and

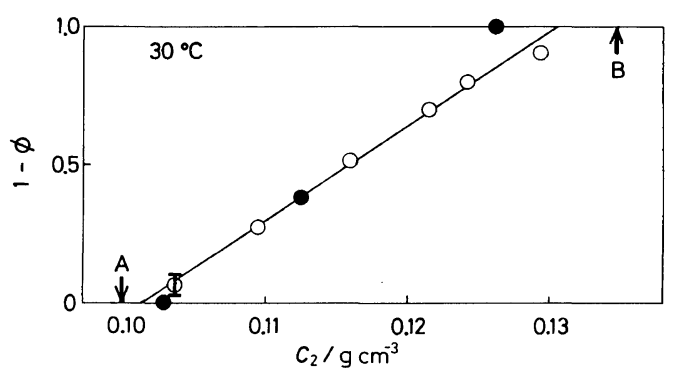

Figure 5. Volume $1-\Phi$ of the cholesteric phase relative to the total solution volume plotted against the overall mass concentration $c_{2}{ }^{0}$ for sample D-40 at $30^{\circ} \mathrm{C}$. Filled circles, data from a separate experiment at $25^{\circ} \mathrm{C}$ (see text); the arrows $\mathrm{A}$ and $\mathrm{B}$, the phase bundary concentrations found in Figure 4. $c_{2}$ in the biphasic region stands for $c_{2}{ }^{0}$.

$3000 \mathrm{rpm}$ were regarded as not being disturbed by the centrifugal force. At these rotor speeds, the redistribution of the polymer species in either phase was almost negligible.

If it is assumed that the phase separation takes place according to the lever rule as in a binary solution, $\Phi$ should be linearly related to the overall mass concentration $c_{2}{ }^{0}$ of the polymer by

$$
1-\Phi=\left(c_{2}{ }^{0}-c_{2}\right) /\left(c_{2}{ }^{\prime}-c_{2}\right)
$$

where $c_{2}{ }^{\prime}$ and $c_{2}$ denote the mass concentrations corresponding to the B-point and A-point, respectively. Recently, Flory and Frost ${ }^{16,17}$ investigated theoretically biphasic equilibria in athermal solutions of polydisperse rodlike particles. It can be 
shown that their numerical results, when recast in the form of eq 2 , predict $\Phi$ decreasing approximately linearly with $c_{2}{ }^{0}$ for a sample with the Poisson distribution in particle size. Samples having the most probable distribution do not obey eq 2 . In the case of the Gaussian distribution, eq 2 holds if the breadth of the distribution is not large. ${ }^{18}$ Thus we may use this equation to analyze experimental phase separation data for polydisperse samples which are not too broad in molecular weight distribution. Indeed, Conio et al. ${ }^{14}$ applied eq 2 to their phase separation data for poly(1,4-benzamide) solutions.

Figure 5 shows a plot of $1-\Phi v s . c_{2}{ }^{0}$ for sample D- 40 at $30^{\circ} \mathrm{C}$. The data points represented by open circles are scattered around the solid line which may be extrapolated to $1-\Phi=0$ and 1 to give $c_{2}=0.101_{2}$ $\mathrm{g} \mathrm{cm}^{-3}$ and $c_{2}{ }^{\prime}=0.130_{5} \mathrm{~g} \mathrm{~cm}^{-3}$, respectively. These values may be compared favorably with those found on the phase diagram (indicated by arrows $\mathrm{A}$ and B). Thus we see that the lever rule holds at least approximately for our schizophyllan + water system, although the sample is not monodisperse.

The filled circle in the middle of the biphasic region represents the data point from a particular experiment conducted at $25^{\circ} \mathrm{C}$, where a somewhat large amount of a solution was separated by centrifugation and the separated solutions were analyzed for concentration and molecular weight. The remaining two filled circles represent the mass concentrations of the separated solutions and are located close to the phase boundaries but not exactly on them. It was found that the molecular weight of the polymer recovered from the isotropic phase was smaller than that of the polymer from the cholesteric phase. This finding is in harmony with the theoretical predictions for athermal solutions of polydisperse rodlike polymers. ${ }^{16-19}$ Similar fractionation effects have been reported for other polymer-solvent systems. ${ }^{12-15}$

\section{DISCUSSION}

The phase diagram in Figure 4 has a narrow biphasic region separating the isotropic region from the cholesteric region and resembles those reported by Miller and coworkers ${ }^{10,11,20}$ for poly $(\gamma$-benzyl Lglutamate) in dimethylformamide, except that it has no broad biphasic region at the bottom. It can be seen that the phase boundary curves are almost vertical at lower temperature but bend toward a higher concentration at higher temperature. A similar trend has also been found for the polypeptide solutions, and is interpreted in terms of the fact that the polypeptide helix becomes more flexible as the temperature is raised. ${ }^{10,11}$ Figure 6 shows the temperature dependence of intrinsic viscosity of schizophyllan D-40 in water, which suggests that the molecular dimensions of schizophyllan decrease with increasing temperature. Thus, we tentatively ascribe the bend of the phase boundary curves in Figure 4 to this increasing flexibility at high temperature, as in the case of the polypeptide solutions.

Various theories ${ }^{21-26}$ have been proposed to describe the phase equilibrium between isotropic and anisotropic phases in a solution of rodlike

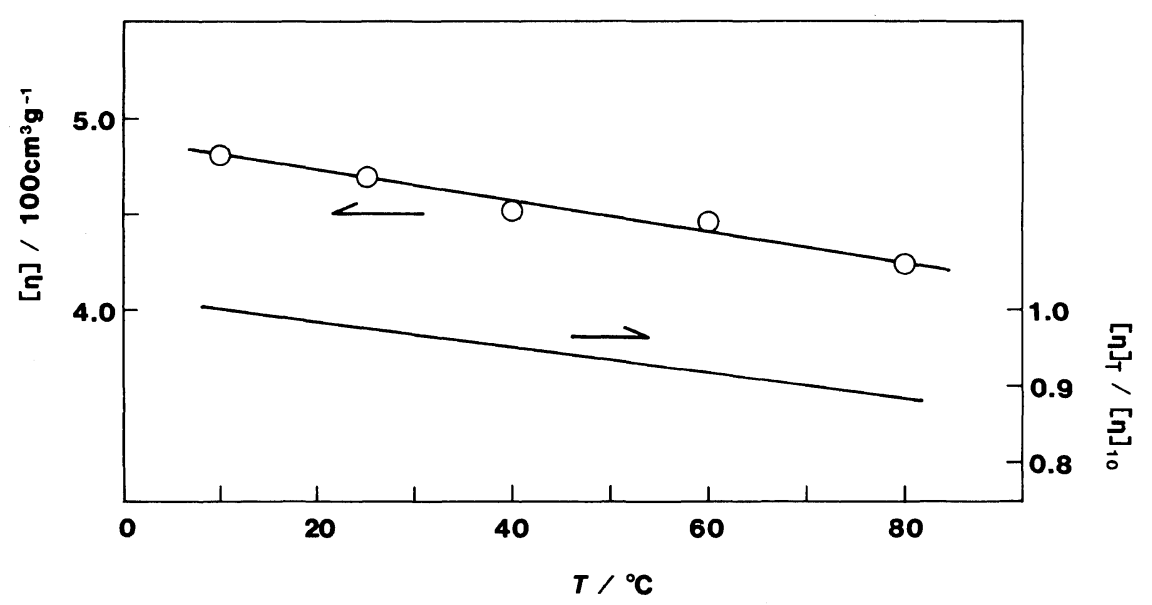

Figure 6. Temperature dependence of intrinsic viscosity for sample D-40 in water. 
Table I. Phase boundary concentrations for the system schizophyllan

D- 40 + water at $25^{\circ}$

\begin{tabular}{lcc}
\hline & $\begin{array}{c}\text { Isotropic } \\
\text { phase }\end{array}$ & $\begin{array}{c}\text { Anisotropic } \\
\text { phase }\end{array}$ \\
\hline & Theoretical & $v_{2}(x=133)$ \\
Onsager $^{21}$ & 0.025 & 0.0334 \\
Flory-Ronca $^{26}$ & 0.0596 & 0.0819 \\
& Experimental & $w_{2}$ and $v_{2}$ \\
$w_{2}$ & 0.0965 & 0.128 \\
$v_{2}$ & 0.062 & 0.0828 \\
\hline
\end{tabular}

polymers. Basically, they are formulated in terms of the volume fractions of the polymer and solvent components, the axial ratio $x$ of the polymer component, and the interaction parameter $\chi$.

For a theoretical analysis of the present phase equilibrium data, we assume that our schizophyllan solutions be athermal and put $\chi=0$, which appears to be a reasonable first approximation compatible with the almost vertical phase boundary curves in Figure 4. This leaves us with only one molecular parameter $x$, which was calculated to be 133 for sample D-40 from $M_{\mathrm{L}}=2140 \mathrm{~nm}^{-1}$ and $\bar{v}^{0}=0.619$ $\mathrm{cm}^{3} \mathrm{~g}^{-1}$, where $M_{\mathrm{L}}$ and $\bar{v}^{0}$ are respectively the molecular weight per unit length and the partial specific volume at infinite dilution of the helix;, ${ }^{2,3}$ the corresponding helix diameter is $1.67 \mathrm{~nm}$. Table I gives the boundary volume fractions calculated for $x=133$ by the theories of athermal solutions. ${ }^{21,26}$ Cotter's theory, ${ }^{25}$ an extension of the Onsager theory ${ }^{21}$ to higher polymer concentrations, predicts an A-point concentration slightly higher than that in the Onsager theory and a narrower biphasic gap.

The experimental phase boundary concentrations obtained in terms of weight fraction were converted to volume fraction $v_{2}$ (or $v_{2}{ }^{\prime}$ ) using eq 1 along with the definition of volume fraction: $v_{2}=w_{2} \bar{v}^{0} / v$. The results are compared with the theoretical predictions in Table I. It can be seen that the Onsager theory predicts too small boundary volume fractions in comparison with those observed. The same was the case with Cotter's theory. On the other hand, the prediction by the Flory-Ronca theory ${ }^{26}$ is close to the experimental observations.

The axial ratio of a rodlike polymer may also be estimated from the second virial coefficient using an appropriate theory so that it conforms to the athermal assumption. Indeed, Kubo and Ogino ${ }^{27,28}$ used in this way the Cotter theory to analyze osmotic pressure and isopiestic data for $\operatorname{poly}(\gamma$-benzyl $\mathbf{L}$ glutamate) in dimethylformamide. They found good agreement between theory and experiment with the data for isotropic solutions of relatively low molecular weight samples. However, the agreement was not satisfactory for anisotropic solutions and for larger molecular weight samples.

It should be remarked that our schizophyllan solution is not strictly an athermal solution consisting of hard rods as the theories require; the second virial coefficient for this system is not exactly consistent with this assumption, yielding a helix diameter of $1.2 \mathrm{~nm},{ }^{29}$ which is much smaller than those estimated from the partial specific volume or hydrodynamic properties of schizophyllan, 1.67$2.6 \mathrm{~nm}^{2-4}$ Generally speaking, the phase relationship for a solution of rodlike polymers is expected to depend on both molecular asymmetry and intermolecular interaction. The molecular asymmetry may be varied using polymer samples of different molecular weights, while information about the intermolecular interaction may be obtained from thermodynamic data for the system concerned. Kubo and Ogino ${ }^{27,28}$ have considered both of these factors in analyzing polypeptide data with partial success. Since we neglected the effects from the intermolecular interaction, the excellent agreement between theory and experiment must be taken with some reservation until phase relationship and thermodynamic data covering the necessary ranges of molecular weight and concentration become available. An experimental study in this direction is now in progress.

Acknowledgment. It is a pleasure to acknowledge the partial support of Taito Co. for this study.

\section{REFERENCES}

1. K. Van, T. Norisuye, and A. Teramoto, Mol. Cryst. Liq. Cryst., 78, 123 (1981).

2. T. Norisuye, T. Yanaki, and H. Fujita, J. Polym. Sci. Polym. Phys. Ed., 18, 547 (1980).

3. T. Yanaki, T. Norisuye, and H. Fujita, Macromolecules, 13, 1462 (1980).

4. Y. Kashiwagi, T. Norisuye, and H. Fujita, Macromolecules, 14, 1220 (1981).

5. W. G. Miller, Ann. Rev. Phys. Chem., 29, 519 (1978).

6. P. W. Morgan, Macromolecules, 10, 1381 (1977).

7. G. C. Berry, P. Metzger, and S. G. Chu, Proceedings 
of U.S.-China Bilateral Symposium on Chemistry and Physics of Polymers, Peking, 1979, in press.

8. G. Maret, M. Milas, and M. Rinaudo, Polym. Bull., 4, 291 (1981).

9. C. Robinson, J. C. Ward, and R. B. Beevers, Discuss. Faraday Soc., 25, 29 (1958).

10. W. G. Miller, J. H. Rai, and E. L. Wee, "Liquid Crystals and Ordered Fluids," Vol. II, R. Porter and J. Johnson, Ed., Plenum, New York, 1974, p 243.

11. W. G. Miller, C. C. Wu, E. L. Wee, G. L. Santee, J. H. Rai, and K. G. Goebel, Pure Appl. Chem., 38, 37 (1974).

12. S. L. Kwolek, P. W. Morgan, J. R. Schaefgen, and L. W. Gulrich, Macromolecules, 10, 1390 (1977).

13. C. Balbi, E. Bianchi, A. Ciferri, A. Tealdi, and W. R. Krigbaum, J. Polym. Sci., Polym. Phys. Ed., 18, 2037 (1980).

14. G. Conio, E. Bianchi, A. Ciferri, and A. Tealdi, Macromolecules, 14, 1084 (1981).

15. S. M. Aharoni and E. K. Walsh, Macromolecules, 12, 271 (1979).

16. P. J. Flory and R. S. Frost, Macromolecules, 11, 1126 (1978).

17. R. S. Frost and P. J. Flory, Macromolecules, 11, 1134
(1978).

18. J. K. Moscicki and G. Williams, Polymer, 23, 558 (1982).

19. A. Abe and P. J. Flory, Macromolecules, 11, 1122 (1978).

20. E. L. Wee and W. G. Miller, J. Phys. Chem., 75, 1446 (1971).

21. L. Onsager, Ann. N. Y. Acad. Sci., 51, 627 (1947).

22. A. Ishihara, J. Chem. Phys., 19, 1142 (1951).

23. P. J. Flory, Proc. R. Soc., London, Ser. A, 234, 73 (1956).

24. R. Alben, Mol. Cryst. Liq. Cryst., 13, 193 (1971).

25. M. A. Cotter, J. Chem. Phys., 66, 1098 (1977).

26. P. J. Flory and G. Ronca, Mol. Cryst. Liq. Cryst., 54, 289 (1979).

27. K. Kubo and K. Ogino, Mol. Cryst. Liq. Cryst., 53, 207 (1979).

28. K. Kubo, Mol. Cryst. Liq. Cryst., 74, 71 (1981).

29. The average value of the second virial coefficient for schizophyllan in water at $25^{\circ} \mathrm{C}$ was found to be $1.25 \times 10^{-4} \mathrm{~mol} \mathrm{~cm} \mathrm{~cm}^{-2}$. $^{3}$ The helix diameter was calculated from this value along with $\vec{v}^{0}$ and $M_{\mathrm{L}}$ values given above by using the theory of Zimm. ${ }^{30}$

30. B. H. Zimm, J. Chem. Phys., 14, 164 (1946). 